\title{
Inverse Spectral Problems in Riemannian Geometry
}

\author{
Peter A. Perry
}

Department of Mathematics, University of Kentucky, Lexington, Kentucky 40506-0027, U.S.A.

\section{Introduction}

Over twenty years ago, Marc Kac posed what is arguably one of the simplest inverse problems in pure mathematics: "Can one hear the shape of a drum?" [19]. Mathematically, the question is formulated as follows. Let $\Omega$ be a simply connected, plane domain (the drumhead) bounded by a smooth curve $\gamma$, and consider the wave equation on $\Omega$ with Dirichlet boundary condition on $\gamma$ (the drumhead is clamped at the boundary):

$$
\begin{array}{cc}
\Delta u(x, t)=\frac{1}{c^{2}} u_{t t}(x, t) & \text { in } \Omega, \\
u(x, t)=0 & \text { on } \gamma .
\end{array}
$$

The function $u(x, t)$ is the displacement of the drumhead, as it vibrates, at position $x$ and time $t$. Looking for solutions of the form $u(x, t)=\operatorname{Re} e^{i \omega t} v(x)$ (normal modes) leads to an eigenvalue problem for the Dirichlet Laplacian on $\Omega$ :

$$
\begin{aligned}
\Delta v(x)+\lambda v(x) & =0 \text { in } \Omega \\
v(x) & =0 \text { on } \gamma
\end{aligned}
$$

where $\lambda=\omega^{2} / c^{2}$. We write the infinite sequence of Dirichlet eigenvalues for this problem as $\left\{\lambda_{n}(\Omega)\right\}_{n=1}^{\infty}$, or simply $\left\{\lambda_{n}\right\}_{n=1}^{\infty}$ if the choice of domain $\Omega$ is clear in context. Kac's question means the following: is it possible to distinguish "drums" $\Omega_{1}$ and $\Omega_{2}$ with distinct (modulo isometries) bounding curves $\gamma_{1}$ and $\gamma_{2}$, simply by "hearing" all of the eigenvalues of the Dirichlet Laplacian?

Another way of asking the question is this. What is the geometric content of the eigenvalues of the Laplacian? Is there sufficient geometric information to determine the bounding curve $\gamma$ uniquely? In what follows we will call two domains isospectral if all of their Dirichlet eigenvalues are the same. We refer to the set of all domains (modulo rigid motions in the plane) with the same Dirichlet eigenvalues as a given domain $\Omega$ as the isaspectral set of $\Omega$. We would like to characterize the isospectral set of a given domain. 
Some surprising and interesting results are obtained by considering the heat equation on $\Omega$ with Dirichlet boundary conditions, which gives rise to the same boundary value problem as before. The heat equation is

$$
\begin{array}{rlrl}
\Delta u(x, t) & =u_{t}(x, t) & & \text { in } \Omega \\
u(x, t) & =0 & & \text { on } \gamma \\
u(x, 0) & =f(x) &
\end{array}
$$

where $u(x, t)$ is the temperature at point $x$ and time $t$, and $f(x)$ is the initial temperature distribution. This evolution equation has the formal solution

$$
u(x, t)=\left(e^{t \Delta} f\right)(x)
$$

where the operator $e^{t \Delta}$ can be calculated using the spectral resolution of $\Delta$. Indeed, if $\phi_{j}(x)$ is the normalized eigenfunction of the boundary value problem (1) with eigenvalue $\lambda_{j}$, the operator $e^{t \Delta}$ has integral kernel $K(t, x, y)$ (the "heat kernel") given by

$$
K(t, x, y)=\sum_{j=1}^{\infty} e^{-t \lambda_{j}} \phi_{j}(x) \phi_{j}(y) .
$$

The trace of $K(t, x, y)$ is actually a spectral invariant: by (2), we can compute

$$
\int_{\Omega} K(x, x, t) d x=\sum_{j=1}^{\infty} e^{-t \lambda_{j}}
$$

Note that the function (3) determines the spectrum $\left\{\lambda_{n}\right\}_{n=1}^{\infty}$.

To analyze the geometric content of the spectrum, one calculates the same trace by a completely different method: one constructs the heat kernel by perturbation from the explicit heat kernel for the plane, and then one computes the trace explicitly. It turns out that the trace has a small- $t$ asymptotic expansion

$$
\int_{\Omega} K(x, x, t) d x \sim \frac{1}{4 \pi t}\left(a_{0}+a_{1 / 2} t^{1 / 2}+a_{1} t+\cdots\right),
$$

where

$$
\begin{aligned}
& a_{0}=\operatorname{area}(\Omega) \\
& a_{1}=\operatorname{length}(\gamma)
\end{aligned}
$$

Although a strict derivation is a bit involved (see Chavel [12] and references therein), there is a simple heuristic argument, due to Mark Kac [19], which shows why $a_{0}$ and $a_{1}$ should give the area of $\Omega$ and the length of $\gamma$. The heat kernel in the plane is

$$
K_{0}(x, y, t)=\frac{1}{4 \pi t} \exp \left(|x-y|^{2} / 4 \pi t\right) .
$$


We expect that, for small times, $K(x, x, t) \simeq K_{0}(x, x, t)$ (a Brownian particle starting out in the interior doesn't "see" the boundary for a time of order $\sqrt{t}$ ) so that, to lowest order,

$$
\int_{\Omega} K(x, x, t) d x \simeq \int_{\Omega} K_{0}(x, x, t) d x=\frac{1}{4 \pi t} \operatorname{area}(\Omega) .
$$

For times of order $\sqrt{t}$, boundary effects become important. We can approximate the heat kernel near the boundary locally by the "method of images." Locally, the boundary looks like the line $x_{1}=0$ in the $x_{1}-x_{2}$ plane; letting $x \mapsto x^{*}$ be the reflection $\left(x_{1}, x_{2}\right) \mapsto\left(-x_{1}, x_{2}\right)$, the kernel

$$
K_{\Omega}(x, y, t)=K_{0}(x, y, t)-K_{0}\left(x, y^{*}, t\right)
$$

vanishes on $x_{1}=0$. Hence,

$$
K_{\Omega}(x, x, t) \simeq \frac{1-e^{-2 \delta^{2} / t}}{4 \pi t}
$$

where $\delta$ is the distance from $x$ to the boundary. Writing the volume integral for the additional term as an integral over the boundary curve and the distance from the boundary,

$$
\int_{\gamma} \int_{0}^{\infty} \frac{1}{4 \pi t} e^{-2 \delta^{2} / t} d \delta d s
$$

we have

$$
\int_{\Omega} K(x, x, t) d x \simeq \frac{\operatorname{area}(\Omega)}{4 \pi t}-\frac{\operatorname{length}(\gamma)}{4} \frac{1}{\sqrt{2 \pi t}}+o\left(\frac{1}{\sqrt{t}}\right) .
$$

It follows that the isospectral set of a given "drum" $\Omega$ contains only drums with the same area and perimeter.

\section{Can one hear the shape of a manifold?}

Kac's early observations stimulated a flurry of work in which mathematicians generalized Kac's problem, studied the geometric content of the spectrum, and constructed counterexamples to Kac's conjecture as generalized to Riemannian manifolds. Guides to the extensive literature in spectral geometry include the books of of Bérard [2], Berger, Gauduchon, and Mazet [3], and Chavel [12].

Here we will briefly discuss the generalization of Kac's problem and some of the known results. A Riemannian manifold of dimension $n$ is a smooth $n$ dimensional manifold $M$ equipped with a Riemannian metric $g$ which defines the length of tangent vectors and determines distances and angles on the manifold. The metric also determines the Riemann curvature tensor of $M$. In two dimensions, the Riemann curvature tensor is in turn determined by the scalar curvature, and in three dimensions it is completely determined by the Ricci curvature tensor. If $M$ is compact, the associated Laplacian has an infinite set of 
discrete eigenvalues $\left\{\lambda_{n}\right\}_{n}=1^{\infty}$. What is the geometric content of the spectrum for a compact Riemannian manifold?

An early paper of Milnor [20] constructs a pair of 16-dimensional torii with the same spectrum. The torii $T_{1}^{n}$ and $T_{2}^{n}$ are quotients of $\mathbf{R}^{n}$ by lattices $\Gamma_{1}$ and $\Gamma_{2}$ of translations of $\mathbf{R}^{n}$. Since the two torii are isometric if and only if their lattices are congruent, it suffices to construct a pair of non-congruent 16dimensional lattices whose associated torii have the same spectrum.

To understand the analysis involved in Milnor's construction, consider the following simple 'trace formula' for a torus $T^{n}=\mathbf{R}^{n} / \Gamma$ which computes the trace of the heat kernel on a torus in terms of the lengths of the lattice vectors of $\Gamma$. Using the "method of images," it is easy to see that the heat kernel on the torus is given by the formula

$$
K_{\Gamma}(x, y, t)=\sum_{\omega \in \Gamma} K_{0}(x+\omega, y, t),
$$

where

$$
K_{0}(x, y, t)=\frac{1}{(4 \pi t)^{n / 2}} e^{-|x-y|^{2} / 4 t}
$$

is the heat kernel on $\mathbf{R}^{n}$. It follows that

$$
\int_{T^{n}} K(x, x, t) d x=\frac{\operatorname{vol}\left(T^{n}\right)}{4 \pi t} \sum_{\omega \in \Gamma} e^{-|\omega|^{2} / 4 t} .
$$

Milnor noted that there exist non-congruent lattices in 16 dimensions with the same set of "lengths" $\{|\omega|: \omega \in \Gamma\}$, first discovered by Witt [26]. Since the trace of the heat kernel determines the spectrum, and the heat trace is in turn determined by the lengths, it follows that the corresponding non-isometric torii have the same spectrum.

Later, other mathematicians found lower-dimensional examples of pairs of non-isometric Riemannian manifolds with the same spectrum. The construction of these examples involved Riemann surfaces with constant curvature and genus $g \geq 5$, and Riemann surfaces with variable curvature and genus $g \geq 3$. Among mathematicians contributing to this research were Vigneraas [25], Buser [4], Sunada [24], and Brooks-Tse [9]. Sunada [24] proved a simple trace formula that reduces the construction of such examples to an exercise in group theory and used it to construct isospectral surfaces. A very readable exposition of this work may be found in the paper of Brooks [6].

All of these examples showed that it was possible to construct pairs (or more generally, finite families) of Riemannian manifolds with the same spectrum. Later, Gordon and Wilson [18] and DeTurck and Gordon [14, 15, 16] constructed continuous families of isospectral manifolds in sufficiently high dimension $(n \geq$ 5). Two major questions remained:

1) Can one show that the isospectral set of a given manifold is at most finite in "low" dimension?

2) Can one find counterexamples for Kac's original problem, i.e., can one construct isospectral, non-congruent planar domains? 
As we will see, some partial progress has been made on the first question and decisive progress has been made on the second.

\section{Some Positive Results}

In 1986, Osgood, Phillips, and Sarnak proved one of the first major positive results on isospectral sets of surfaces and planar domains. Informally, a sequence of planar domains $\Omega_{j}$ converges in $C^{\infty}$ sense to a limiting, non-degenerate domain $\Omega$ if the bounding curves $\gamma_{j}$ converge in $C^{\infty}$ sense modulo rigid motions of the plane and the limit curve encloses a nondegenerate bounded region. Similarly, a set of compact surfaces $S_{j}$ converges in $C^{\infty}$ sense to a limiting, non-degenerate surface $S$ if the surfaces are all diffeomorphic to $S$ and the metrics $g_{j}$ on $S_{j}$, pulled back to $S$, converge in $C^{\infty}$ sense to a positive definite metric on $S$. Osgood, Phillips, and Sarnak showed [22]:

Theorem 1. (i) Let $\Omega_{j}$ be a sequence of isospectral planar domains. There is a subsequence which converges in $C^{\infty}$ sense to a nondegenerate limiting surface.

(ii) Let $S_{j}$ be a sequence of isospectral compact surfaces. There is a subsequence of the $S_{j}$ converging in $C^{\infty}$ sense to a non-degenerate surface $S$.

Later, Osgood, Phillips, and Sarnak [23] generalized the first part of their result to non-simply connected planar domains.

If one can show that the isospectral set of a given domain or surface is discrete, the compactness result implies that the isospectral set of the given domain or surface is finite. Although no such discreteness results have yet been proved, there has been some progress studying the isospectral sets of Riemannian manifolds in higher dimensions. Brooks, Perry, and Yang [7] and Chang and Yang $[10,11]$ studied isospectral sets of conformally equivalent metrics on a fixed compact manifold in three dimensions: that is, metrics on a fixed underlying smooth manifold which define the same angles, but not necessarily the same distances. Later, Anderson [1] and Brooks, Perry, and Petersen [8] studied sets of manifolds with the same spectrum and were able to prove compactness subject to certain a priori assumptions on the geometry. Brooks, Perry, and Petersen proved:

Theorem 2. Suppose $\left\{M_{j}\right\}$ is a sequence of isospectral manifolds such that either

(i) All of the $M_{j}$ have negative sectional curvatures, or

(ii) All of the $M_{j}$ have Ricci curvature bounded below

Then there are finitely many diffeomorphism types, and there is a subsequence which converges in $C^{\infty}$ to a nondegenerate limiting manifold.

It is expected that the curvature constraints can be removed eventually. 
An important role is played in both of these theorems by two kinds of spectral invariants: local invariants expressible as integrals of the curvature tensor and its derivatives over the domain or manifold and non-local invariants such as the determinant of the Laplacian and the eigenvalues themselves.

Let us consider the case of a compact manifold $M$ without boundary in 2 or 3 dimensions. Typically, the local invariants are the heat invariants arising in the small-t asymptotic expansion of the heat trace. If $K_{M}(x, y, t)$ is the fundamental solution kernel for the heat equation on a Riemannian manifold $M$, one has the asymptotic expansion

$$
\int_{M} K(x, x, t) d \operatorname{vol}(x) \sim(4 \pi t)^{-n / 2} \sum_{j=0}^{\infty} a_{j} t^{j}
$$

with the $a_{j}$ spectral invariants. The term $a_{0}$ gives the volume of the Riemannian manifold, and the higher-order terms are integrals over $M$ of the scalar $(n=2)$ or Ricci $(n=3)$ curvatures and their covariant derivatives up to order $j-2$.

The heat invariants by themselves do not contain sufficient information to prove compactness. An easy way to see this is to note that there are deformations of constant curvature surfaces of genus 2 or higher in which the length of a closed geodesic tends to zero, thereby dividing the manifold into two parts in the limit as $\ell \rightarrow 0$ (see figure 1). Since the heat invariants in this case depend on the scalar curvature and its derivatives, $a_{0}$ is constant and the remaining invariants are all zero! Geometrically, the quantity which becomes uncontrolled in the limit $\ell \rightarrow 0$ is the diameter of the manifold, defined as the maximum of $\operatorname{dist}_{M}(x, y)$ where $\operatorname{dist}_{M}(\cdot, \cdot)$ is the distance with respect to the Riemannian metric on $M$.

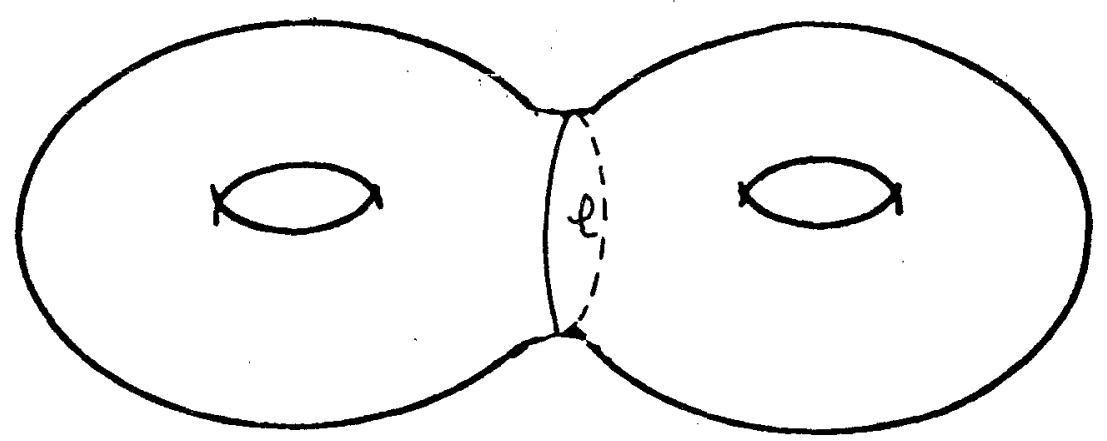

Fig. 1.

What are the nonlocal invariants which prevent degeneration of isospectral manifolds? Osgood, Phillips and Sarnak studied the determinant of the Laplacian, a renormalized product of the nonzero eigenvalues of the Laplacian which is manifestly a spectral invariant. Using results of Wolpert [27], they showed 
that, for degenerations of the type described above, $\operatorname{det}(-\Delta) \rightarrow 0$ as $\ell \rightarrow 0$, so that fixity of the determinant of the Laplacian rules out such degenerations for isospectral surfaces.

In three dimensions, nonlocal invariants are again used to control the diameter of isospectral manifolds. Cheng's comparison theorem [13] implies that, given a bound on Ricci curvature, one can show that the spectrum bounds the diameter from above (see [8]). Intuitively, if the diameter of the manifold is too large, one can construct test functions in a large number of geodesic balls and obtain a contradiction use the max-min principle. More generally, one expects blow-up of the diameter or other singularity formation to lead to concentration of eigenvalues forbidden by isospectrality.

\section{Counterexamples Revisited}

Perhaps the most remarkable recent development relating to Kac's problem is the construction, by Gordon, Webb, and Wolpert [17], of isospectral, nonisometric planar domains (figure 2). These domains are obtained from isospectral manifolds-with-boundary constructed using the Sunada technique [24]. One can actually prove isospectrality by the so-called method of transplantation: one can take an eigenfunction of the first domain $\Omega_{1}$, restrict it to each of the triangles $A-G$, and rearrange the pieces in the second domain as shown. Since the new functions satisfy the Dirichlet boundary conditions and are continuous along each triangle boundary, they are eigenfunctions of the Laplacian on $\Omega_{2}$. This shows that the Dirichlet spectrum of $\Omega_{1}$ is contained in the Dirichlet spectrum of $\Omega_{2}$. A similar transplantation argument shows the reverse inciusion.

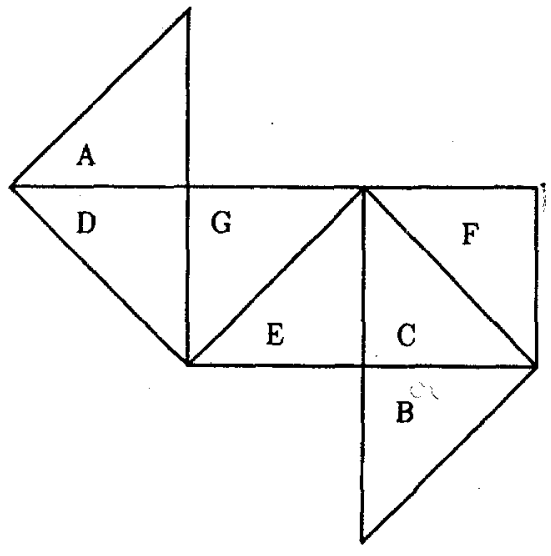

Domain 1

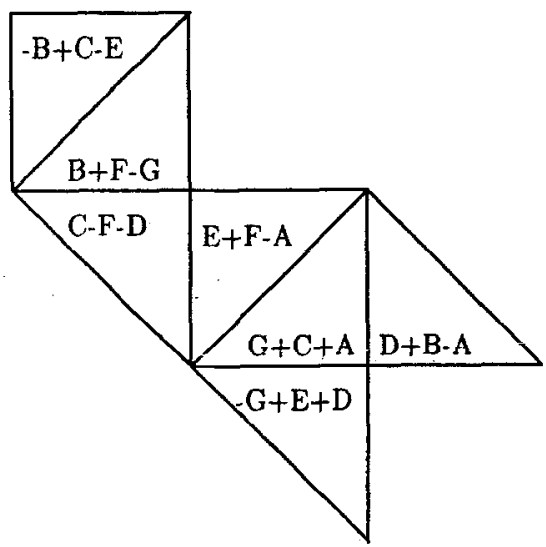

Domain 2

Fig. 2. 
More recently, Buser, Conway, Doyle, and Semmler [5] have shown that one can construct pairs of drums which "sound alike" in the stronger sense that there are points $x_{1} \in \Omega_{1}$ and $x_{2} \in \Omega_{2}$ with the property that if $\Omega_{1}$ is hit at $x_{1}$, it makes exactly the same sound as $\Omega_{2}$ does when hit at $x_{2}$. That is, the solutions to the wave equations in $\Omega_{i}$

$$
\begin{aligned}
\Delta u^{i}-u_{t t}^{i} & =0 \\
u^{i}(x, 0) & =0 \\
u_{t}^{i}(x, 0) & =\delta\left(x-x_{i}\right)
\end{aligned}
$$

with Dirichlet boundary conditions satisfy $u^{1}\left(x_{1}, t\right)=u^{2}\left(x_{2}, t\right)$ for all $t>0$.

Thus, one may say, with apologies to Henry David Thoreau, that it is possible to march to the sound of a different drummer, and hear the same beat!

\section{References}

1. Anderson, M: Remarks on the compactness of isospectral sets in low dimension. Duke Math. J. 63 (1991) 699-711.

2. Bérard, P.: Spectral geometry : Direct and Inverse Problems. Lect. Notes in Math. 1207 (1986).

3. Berger, M., Gauduchon, P., Mazet, E.: Le Spectre d'une Variété Riemannienne. Lect. Notes in Math. 194 (1974).

4. Buser, P.: Isospectral Riemann surfaces. Ann. Inst. Fourier Grenoble 36 (1988) 167-192.

5. Buser, P., Conway, J., Doyle, P., Semmler, E.: Preprint, 1992.

6. Brooks, R.: Constructing isospectral manifolds. Amer. Math. Monthly 95 (1988) 823-839.

7. Brooks, R., Perry, P., Yang, P.: Isospectral sets of conformally equivalent metrics. Duke Math. J. 58 (1989) 131-150.

8. Brooks, R., Perry, P., Petersen P. V.: Compactness and finiteness theorems for isospectral manifolds. J. Reine angew. Math 426 (1992) 67-89.

9. Brooks, R., Tse, R.: Isospectral surfaces of small genus. Nagoya J. Math. 107 (1987) 13-24.

10. Chang, S.-Y. A., Yang, P.: Compactness of isospectral conformal metrics on $S^{3}$. Comment. Math, Helv. 64 (1989) 363-374.

11. Chang, S.-Y. A., Yang, P.: Isospectral conformal metrics on 3-manifolds. J. American Math. Soc. 3 (1990) 117-145.

12. Chavel, I.: Eigenvalues in Riemannian Geometry (Pure and Applied Mathematics, v. 115). New York: Academic Press, 1984.

13. Cheng, S. Y.: Eigenvalue comparison theorems and its geometric applications. Math. Zeits. 143 (1975) 289-297.

14. DeTurck, D., Gordon, C.: Isospectral Riemannian metrics and potentials. Bull. A. M. S. 17 (1987) 137-140.

15. DeTurck, D., Gordon, C.: Isospectral deformations, I. Riemannian structures on two-step nilspaces. Comm. Pure Appl. Math. 40 (1987) 367-387.

16. DeTurck, D., Gordon, C.: Isospectral deformations, II. Trace formulas, metrics, and potentials. Comm. Pure Appl. Math. 42, (1989) 1067-1095. 
17. Gordon, C., Webb, D., Wolpert, S.: You can't hear the shape of a drum. Preprint, 1992.

18. Gordon, C., Wilson, E.: Isospectral deformations of compact solvmanifolds. J. Diff. Geom. 19 (1984) 241-256.

19. Kac. M.: "Can one hear the shape of a drum?" Amer. Math. Monthly 73 (1966) 1-23.

20. Milnor, J.: Eigenvalues of the Laplace operator on certain manifolds. Proc. Nat. Acad. Sci. U. S. A. 51 (1964) 342.

21. Osgood, B., Phillips, R., Sarnak, P.: Extremals of determinants of Laplacians. J. Funct. Anal. 80 (1988) 148-211.

22. Osgood, B., Phillips, R., Sarnak, P.: Compact isospectral sets of surfaces. J. Funct. Anal. 80 (1988) 212-234.

23. Osgood, B., Phillips, R., Sarnak, P.: Moduli space, heights and isospectral sets of plane domains. Ann. of Math. 129 (1989) 293-362.

24. Sunada, T.: Riemannian coverings and isospectral manifolds. Ann. Math. 121 (1984) 169-186.

25. Vignéras, M. F.: Variétés Riemanniennes isospectrales et non isométriques. Ann. Math. 112 (1980) 21-32.

26. Witt, E.: Eine Identität zwischen Modulformen Zweiten Grades. Ab. Math. Sem. Hansischen Univ. 14 (1941) 323-337.

27. Wolpert, S.: Asymptotics of the spectrum and the Selberg zeta function on the space of Riemann surfaces. Commun. Math. Phys. 113 (1987) 283-325. 